\title{
ENHANCED QUALITY NANOCRYSTALLINE METAL FILMS PRODUCED BY DENDRIMER MEDIATED THIN FILM GROWTH
}

\author{
FENGTING XU*,LONG LI*, JUDITH C. YANG*, SHANE C. STREET**, JOHN A. BARNARD* \\ *Department of Materials Science and Engineering, 848 Benedum Hall, University of Pittsburgh, \\ Pittsburgh, PA 15260. \\ **Department of Chemistry, Box 870268, University of Alabama, Tuscaloosa, AL 35487.
}

Dendrimers are three-dimensional, globular, highly-branched macromolecules made up of a central core surrounded by repetitive units all enclosed by a terminal group 'shell'. They can be synthesized with highly controllable sizes (they are monodisperse) determined by the core type, extent of branching, and nature of the end groups, in the range from a few to several tens of nm in diameter $[1,2]$. Dendrimers also assemble into monolayers (Figure 1) on technologically interesting substrates using simple cleaning, dipping, and rinsing procedures. In this 'condensed' monolayer phase dendrimers can act as 'surfactants' mediating the growth of ultraflat films, and create novel nanomechanical, adhesive, frictional, and tribological behavior.

We have recently reported the dramatically enhanced quality (superior flatness and adhesion) of metal films deposited on dendrimer monolayers [3,4]. Figure 1(a) and 1(b) are schematic diagrams of the metal thin film deposited onto a silica/silicon substrate without and with a dendrimer layer, respectively. The improved adhesion and hardness of various dendrimer-mediated metal thin films were determined by nanoscratch tests with an atomic force microscope (AFM) (Figure 2(a) and 2(b)). To understand the substrate/dendrimer monolayer/adlayer interactions and how they determine the physical, mechanical, and chemical properties of the resulting dendrimer-based nanocomposites, we are examining the structure of metal nanocrystalline films formed with and without dendrimers on amorphous silica by transmission electron microscopy (TEM).

Our initial TEM results revealed that the dendrimers significantly alter the grain structure of the metal thin film. Figure 3(a) is a bright field image of the $\sim 10 \mathrm{~nm} \mathrm{Au}$ deposited on $\sim 3 \mathrm{~nm} \mathrm{SiO}_{2}$ which was deposited on a SiN window TEM grid, and figure $3(\mathrm{~b})$ is the corresponding TEM image of $\sim 10 \mathrm{~nm}$ $\mathrm{Au}$ deposited on the dendrimer G8 which was deposited on a similar substrate material. Large grains ( $\sim 5 \mathrm{~nm}$ to $\sim 30 \mathrm{~nm}$ grain size) of $\mathrm{Au}$ was observed on the non-dendrimer substrate, whereas the $\mathrm{Au}$ grains formed on the dendrimer were smaller $(\sim 7-20 \mathrm{~nm})$ and more uniform in distribution. The insets are the corresponding diffraction patterns demonstrating the polycrystalline nature of the Au films. It is reasonable to speculate that the Au thin film composed of smaller and more uniform Au grains should have better mechanical properties than a metal film consisting of larger and non-uniform Au grains. The ability to grow flat, continuous, and conformal ultra-thin $(\sim 1-10 \mathrm{~nm})$ films of metals, oxides, nitrides, etc., is critical to progress in a wide array of advanced technologies including data storage, microelectronics, MEMS, and protective coatings.

\section{References}

[1] D.A. Tomalia, A.M. Naylor, W.A. Goddard, Angew. Chem. 1994 102, 119-157.

[2] D.A. Tomalia, Adv. Mater. 1994, 6, 529-539.

[3] A. Rar, J. Zhou, W.J. Liu, J. Barnard, A. Bennett, and S. Street, Appl. Surf. Sci. 2001, 175, 134-139.

[4] S.C. Street, A. Rar, J.N. Zhou, W.J. Liu, and J.A. Barnard, Chem. Mater. 2001, 13, 3669-3677. 

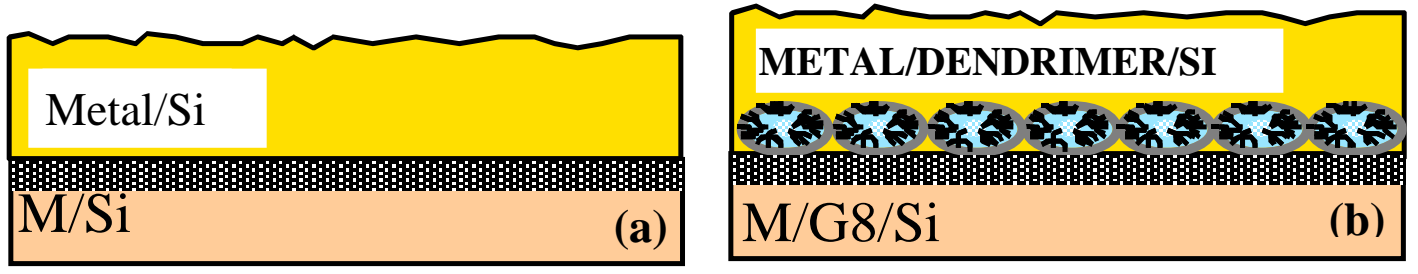

Figure 1. Schematic diagram of the metal, with and without dendrimer(G8), samples deposited on silica/Si substrate. (a) Metal layer without dendrimer, (b) metal layer with dendrimer interlayer.

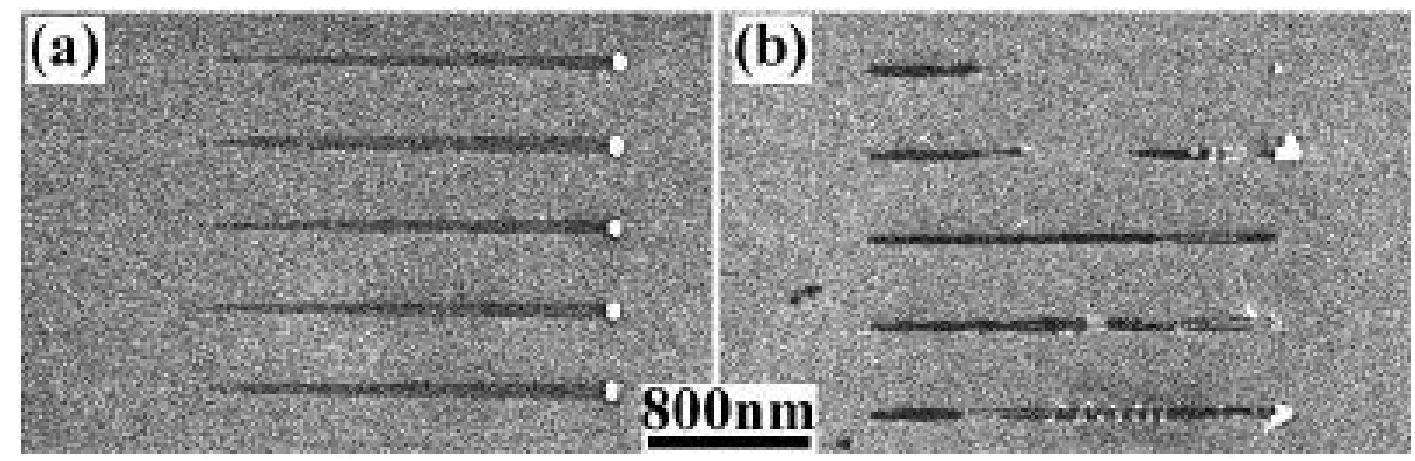

Figure 2. SEM images of five parallel $4 \mathrm{mN}$ constant load scratches on (a) metal $(\mathrm{Cu})$ film without dendrimer, and (b) metal film with G8 dendrimer interlayer.

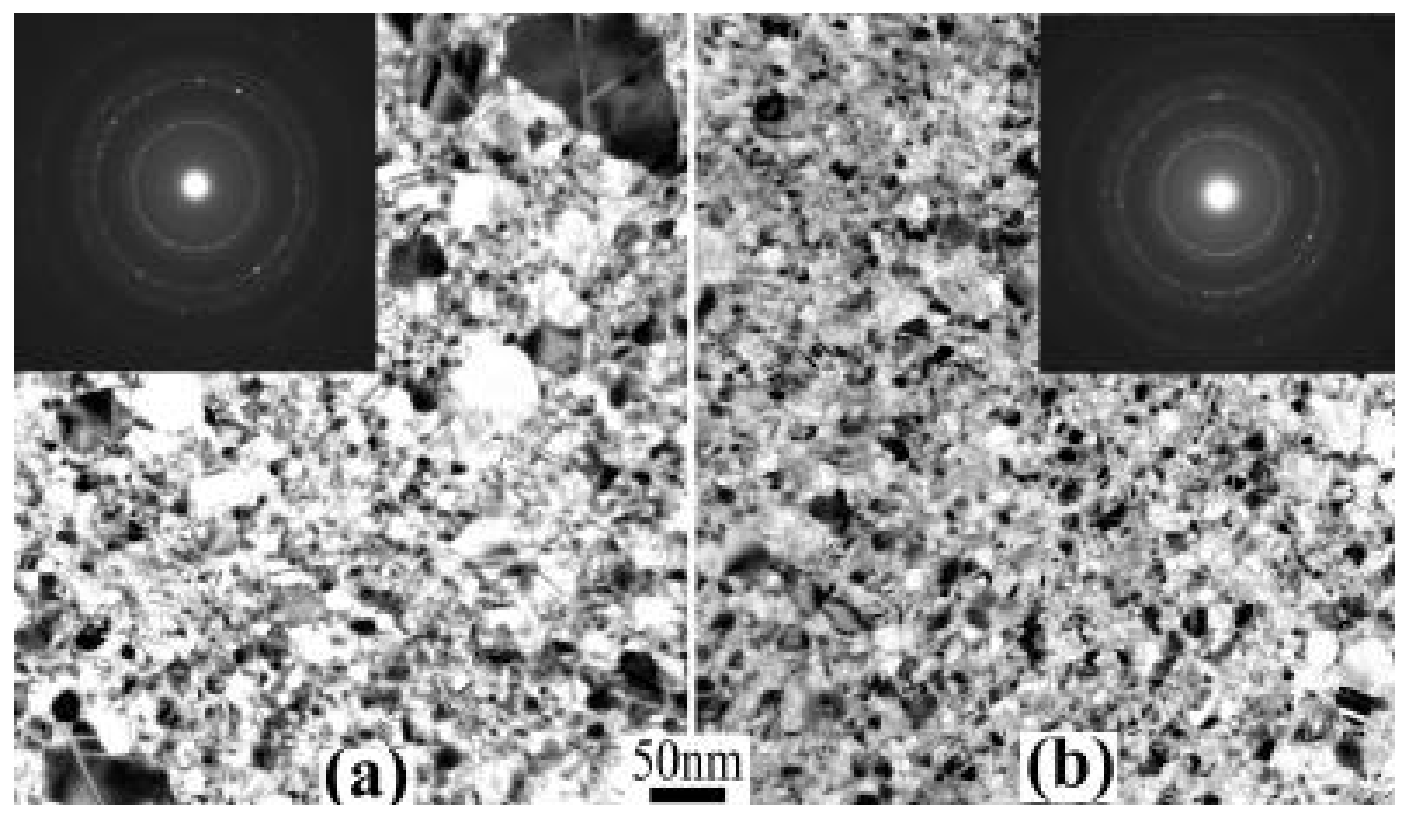

Figure 3. TEM bright field images with corresponding electron diffraction patterns of (a) metal $(\mathrm{Au})$ film without dendrimer, and (b) metal film with G8 dendrimer interlayer. 